\title{
SULLA INFLUENZA DEL FONDO NELLA PROPAGAZIONE DELLE ONDE DOVUTE A PERTURBAZIONI LOCALI. STUDIO ASINTOTICO DEL PELO LIBERO.
}

\author{
Memoria di Attilio Palatini (Padova).
}

Adunanza del 28 novembre r915,

\section{PREFAZIONE.}

In una mia Memoria, pubblicata in questi Rendiconti ${ }^{2}$ ), mi sono proposto di determinare l'influenza del fondo sulla propagazione delle onde in un canale di grande profondità. In tale Memoria ho ricondotto il problema alle quadrature, riuscendo a dare alla espressione del pelo libero la forma di integrale definito. Avendo osservato che le leggi del fenomeno andavano desunte dal comportamento asintotico di quell'integrale, mi sono riservato il compito di uno studio ulteriore dellintegrale medesimo. Il presente lavoro ha lo scopo di desumerne un valore approssimato.

Per tal fine mi varró di un concetto, che inspiró il Riemann per determinare appunto il valore asintotico di un integrale, concetto che ci è pervenuto soltạnto attraverso alcuni brevi frammenti, che dovevano certamente servire al RiEmann stesso per un lavoro "Sullo svolgimento del quoziente di due serie ipergeometriche in frazione continua infinita " ${ }^{2}$ ). Alcuni autori [in ispecie Debye e Brillouin ${ }^{3}$ )] raccolsero il pensiero del Riemann e ne fecero un metodo, che oggi i tedeschi chiamano Methode der Sattelpunkte.

Ricordo dalla prefazione di M. che nell'equazione del pelo libero comparíscono due integrali, di cui uno contiene la profondità del canale e l'altro ne é indipendente e corrisponde al caso di un canale infinitamente profondo. Per l'applicazione del metodo di Riemann sono costretto a dare a questo integrale una forma diversa da quella classica riportata in $M$. Questa nuova forma coincide sostanzialmente con quella assegnatale

I) A. Palatini, Sulla influenza del fondo nella propagazione delle onde dovute a perturbazioni locali [Rendiconti del Circolo Matematico di Palermo, t. XXXIX (I ${ }^{\circ}$ semestre I9I5), pp. 362-384]. Questa Memoria sarà indicata nel presente lavoro con $M$.

2) B. Riemann, Gesammelte mathematische Werke (Leipzig, Teubner, I876), p. 400.

3) P. DeBYe, Näherungsformeln für die Zylinderfunktionen [Mathematische Annalen, Band LXIV (1909), pp. 535-558]; L. Brillouin, Die Fortpflanzug des Lichtes in dispergierenden Medien [Annalen der Physik, Band XLIV (I9I4), pp. 203-240]. 
da CAUChY, che però il grande geometra scrive in modo che, per il rigore matematico odierno, viene ritenuto scorretto. Io quindi, per ottenerla, mi varrò esclusivamente delle mie formule e la scriveró in modo un poco diverso. A questa trasformazione è dedicato il $\mathrm{I}^{0} \int$ di questa Memoria.

Ho esposto poi brevemente, per quello che mi è strettamente necessario, il concetto che presiede al metodo di Riemann, rimandando cosi il lettore, per la conoscenza completa del metodo stesso, alle citate Memorie di Brillouin e Debye.

Infine, applicando il metodo di RiEmanN, sono passato allo studio asintotico del pelo libero.

\section{$\int \mathrm{I}$.}

\section{La nuova espressione di $n$.}

I. Richiamo alla precedente Memoria, - Poichè le considerazioni che andremo svolgendo in questo primo $\$ hanno interesse puramente analitico, richiameremo il più brevemente possibile quanto ci è necessario per collegare le presenti ricerche con la precedente Memoria.

Ricordiamo che, assumendo come unità di lunghezza la profonditì $b$ del canale, il campo del moto (nel piano complesso $z=x+i y$ ) è costituito da una striscia piana $L$, compresa tra le due rette $y=0$ e $y=\mathrm{I}$.

Ricordiamo poi che il moto ondoso, che ci interessa studiare, è retto dall'equazione differenziale:

essendo

$$
\frac{\partial^{4} f}{\partial t^{4}}+g^{2} \frac{\partial^{2} f}{\partial z^{2}}=o
$$

$$
f=\psi+i \psi
$$

e denotando con $\varphi$ il potenziale di velocita e con $\psi$ la funzione di corrente.

L'equazione della linea libera è

$$
n=-\frac{I}{g}\left[\frac{\partial \varphi}{\partial t}\right]_{y=1}
$$

[ $n=$ ordinata contata verso l'alto a partire dal livello medio], o, ciò che è lo stesso

$$
n=-\frac{1}{g} \lim _{\varepsilon=1} R\left[\frac{\partial f}{\partial t}\right]_{z=x+i \varepsilon}
$$

che, come vedremo, è più giovevole per lo studio asintotico che abbiamo in vista. Nella (2) $R$ denota il passaggio alla parte reale ed $f$ è l'integrale generale della ( $I$ ). In base alle condizioni iniziali, per la $\frac{\partial f}{\partial t}$ abbiamo trovato la seguente espressione:

(3) $\frac{\partial f}{\partial t}=-a g\left\{\sum_{0}^{\infty}(-1)^{n} \frac{t^{4 n}}{(4 n) !} g^{2 n} \frac{d^{2 n}}{d z^{2 n}} \frac{1}{2 \cos \frac{\pi i z}{2}}-\sum_{0}^{\infty}(-1)^{n} \frac{t^{4 n+2}}{(4 n+2) !^{2 n+1}} \frac{d^{2 n+1}}{d z^{2 n+1}} \frac{z}{2 \cos \frac{\pi i z}{2}}\right\}$. 
Si tratta ora di dare a questa espressione la forma integrale, per poi da questa ottenere l'equazione del pelo libero a norma della (2).

2. Deduzione di alcune formule ausiliarie - Procuriamoci, in primo luogo, alcune relazioni, di cui ci dovremo valere nei successivi paragrafi.

Nel $n^{\circ}$ I del $\int$ III di M., abbiamo ricavato la seguente formula:

$$
f\left(z^{\prime}\right)=\int_{-\infty}^{\infty} \varphi_{y=1} \frac{d x}{e^{\frac{\pi}{2}\left(x-z^{\prime}\right)}+e^{-\frac{\pi}{2}\left(x-x^{\prime}\right)}}
$$

che dà il valore della funzione $f=\varphi+i \downarrow$ della variabile complessa $z=x+i y$, reale sull'asse reale, in un punto qualunque $z^{\prime}$ interno alla striscia compresa fra l'asse reale e la retta $y=\mathrm{I}$, quando sia nota la successione dei valori, che la sua parte reale assume sulla retta $y=\mathrm{I}$. Per la validità della (4) è necessario che la $f$ sia monodroma, finita e continua entro la striscia, ed inoltre tale che il modulo del rapporto $-\frac{\left(z-z^{\prime}\right) f\left(z^{\prime}\right)}{e^{\frac{\pi}{2}\left(z-z^{\prime}\right)}-e^{-\frac{\pi}{2}\left(x-z^{\prime}\right)}}$ tenda a zero, quando il modulo di $\left(z-z^{\prime}\right)$ tende all'infinito.

Ora se $m$ è una quantità reale, la funzione $\cos (m z)$ soddisfa a tutte le condizioni poste per la $f$; ed essendo in questo caso:

$$
\varphi_{y=1}=\cos (m x) \frac{e^{m}+e^{-m}}{2}
$$

la (4) porge, in particolare:

$$
\frac{2 \cos \left(m z^{\prime}\right)}{e^{m}+e^{-m}}=\int_{-\infty}^{\infty} \frac{\cos (m x) d x}{e^{\frac{\pi}{2}\left(x-z^{\prime}\right)}+e^{-\frac{\pi}{2}\left(x-z^{\prime}\right)}}
$$

da cui, per $z^{\prime}=0$ [e chiamando con $\tau$ la variabile corrente di integrazione]:

$$
\frac{I}{e^{m}+e^{-m}}=\int_{e_{0}^{\infty}}^{\infty} \frac{\cos (m \tau) d \tau}{e^{\frac{\pi}{2} \tau}+e^{-\frac{\pi}{2} \tau}} .
$$

Consideriamo ora i due membri della $(5)$ come funzioni della variabile complessa $m$ e chiamiamole per un momento: la $\mathrm{I}^{\mathrm{a}} \chi_{\mathrm{I}}(m)$ e la $\mathrm{II}^{\mathrm{a}} \chi_{2}(m)$. La $\chi_{1}$ è una funzione analitica che esiste per tutti $\mathrm{i}$ valori complessi di $m$ : la $\chi_{2}$ invece è una funzione analitica regolare di $m$, purchè si supponga che il coefficiente di $i$ sia, in valore assoluto, minore di $\frac{\pi}{2}$. E poichè le due funzioni $\chi_{1}$ e $\chi_{2}$ coincidono per valori reali di $m$, coincidono altresi per tutti i valori complessi di $m$ che sono compresi nel campo di comune esistenza. La (5) vale quindi non solo per $m$ reale, ma anche per $m$ complesso, purchè tale che il coefficiente dell'immaginario sia minore di $\frac{\pi}{2}$.

Rappresenti ora $z$ l'affissa di un punto qualunque interno al campo $L$; il coef. 
ficiente di $i$ nel prodotto $\frac{\pi}{2} z$ è certamente minore di $\frac{\pi}{2}$, per cui, posto nella $(5) \frac{\pi}{2} z$ in luogo di $m$, avremo:

$$
\frac{1}{2 \cos \frac{\pi i z}{2}}=\frac{2}{\pi} \int_{0}^{\infty} \frac{\cos (\tau z) d \tau}{e^{\tau}+e^{-\tau}} .
$$

Moltiplicando entrambi i membri della (6) per $z$, eseguendo una integrazione per parti e derivando poscia $i$ due membri rispetto a $z$, si ottiene:

$$
\frac{d}{d z} \frac{z}{2 \cos \frac{\pi i z}{2}}=\frac{2}{\pi} \int_{0}^{\infty} \cos (\tau z) \cdot \tau \frac{e^{\tau}-e^{-\tau}}{\left(e^{\tau}+e^{-\tau}\right)^{2}} d \tau
$$

Mediante una derivazione di ordine $2 n$, si hanno poi dalle (6) e (7) le due seguenti espressioni:

(8)

$$
\left\{\begin{array}{l}
(-\mathrm{I})^{n} \frac{d^{2 n}}{d z^{2 n}}-\frac{1}{2 \cos \frac{\pi i z}{2}}=\frac{2}{\pi} \int_{0}^{\infty} \frac{\cos (\tau z)}{e^{\tau}+e^{-\tau} \tau^{2 n} d \tau} \\
(-\mathrm{I})^{n} \frac{d^{2 n+1}}{d z^{2 n+1}} \frac{z}{2 \cos \frac{\pi i z}{2}}=\frac{2}{\pi} \int_{0}^{\infty} \cos (\tau z) \cdot \tau^{2 n+1} \frac{e^{\tau}-e^{-\tau}}{\left(e^{\tau}+e^{-\tau}\right)^{2}} d \tau .
\end{array}\right.
$$

Osserviamo infine, che, dai noti sviluppi in serie della funzione esponenziale della funzione coseno, si ha:

$$
\left\{\begin{array}{l}
\cosh t \sqrt{g \tau}+\cos t \sqrt{g \tau}=2 \sum_{0}^{\infty} \frac{t^{4 n} g^{2 n} \tau^{2 n}}{(4 n) !}, \\
\cosh t \sqrt{g \tau}-\cos t \sqrt{g \tau}=2 \sum_{0}^{\infty} \frac{t^{4 n+2} g^{2 n+1} \tau^{2 n+1}}{(4 n+2) !} .
\end{array}\right.
$$

3. Passaggio alle quadrature. - Ciò premesso, la (3), con l'aiuto delle (8), assume la forma:

$$
\begin{aligned}
\frac{\partial f}{\partial t}=-a g\left\{\frac{2}{\pi} \sum_{0}^{\infty} \frac{t^{4 n}}{(4 n) !} g^{2 n}\right. & \int_{0}^{\infty} \frac{\cos (\tau z)}{e^{\tau}+e^{-\tau} \tau^{2 n} d \tau} \\
& \left.-\frac{2}{\pi} \sum_{0}^{\infty} \frac{t^{n+2}}{(4 n+2) !} g^{2 n+1} \int_{0}^{\infty} \cos (\tau z) \cdot \tau^{2 n+1} \frac{e^{\tau}-e^{-\tau}}{\left(e^{\tau}+e^{-\tau}\right)^{2}} d \tau\right\}
\end{aligned}
$$

e questa a norma delle (9), si trasforma nella:

$$
\begin{aligned}
\frac{\partial f}{\partial t}=\frac{-a g}{\pi}\left\{\int_{0}^{\infty} \frac{\cos (\tau z)}{e^{\tau}+e^{-\tau}}\right. & {[\cosh t \sqrt{g \tau}+\cos t \sqrt{g \tau}] d \tau } \\
& \left.-\int_{0}^{\infty} \cos (\tau z) \frac{e^{\tau}-e^{-\tau}}{\left(e^{\tau}+e^{-\tau}\right)^{2}}[\cosh t \sqrt{g \tau}-\cos t \sqrt{g \tau}] d \tau\right\} .
\end{aligned}
$$


Questa equazione, in fine, si può scrivere nel modo seguente:

$$
\frac{\partial f}{\partial t}=-\frac{a g}{\pi}\left\{2 \int_{0}^{\infty} \frac{\cos (\tau z) \cos t \sqrt{g \tau}}{e^{\tau}+e^{-\tau}} d \tau+2 \int_{0}^{\infty} \frac{e^{-\tau} \cos (\tau z)}{\left(e^{\tau}+e^{-\tau}\right)^{2}}[\cosh t \sqrt{g \tau}-\cos t \sqrt{g \tau}] d \tau\right\} .
$$

4. Equazione del pelo libero (in unità generiche). - Se ora in questa espressione poniamo $z=x+i \varepsilon$ e separiamo poi il reale dall'immaginario, in virtù della (2), otteniamo immediatamente :

$$
n=\frac{a}{\pi}\left\{\lim _{\varepsilon=1} \int_{0}^{\infty} \cos (\tau x) \cos t \sqrt{g \tau} \frac{e^{\tau \varepsilon}+e^{-\tau \varepsilon}}{e^{\tau}+e^{-\tau}} d \tau+\int_{0}^{\infty} \frac{\cos (\tau x)}{\mathrm{I}+e^{2 \tau}}[\cosh t \sqrt{g \tau}-\cos t \sqrt{g \tau}] d \tau\right\} .
$$

Ricordiamo che fin qui abbiamo scelto come unità di lunghezza la profondità $b$ del canale: otteniamo la corrispondente espressione di $n$ in uniti generiche, alterando tutte le lunghezze nel rapporto $\mathrm{d} a \mathrm{I}$ ad $\mathrm{I} / h$.

Scambiando allora il $\tau$ in $\tau^{2} / x$ (dopo aver supposto, come in M., che $x$ sia positivo) e fatte le seguenti posizioni:

$$
\begin{gathered}
\omega=\frac{g t^{2}}{2 x}, \\
P(\tau, \varepsilon)=\frac{e^{\tau \frac{\varepsilon \varepsilon h}{x}}+e^{-\tau^{2 \frac{\varepsilon}{x}}}}{e^{2 \frac{b}{x}}+e^{-\tau^{2} \frac{b}{x}}}, \\
K=\lim _{\varepsilon=1} 2 \int_{0}^{\infty} \cos \tau^{2} \cos \tau \sqrt{2 \omega} \cdot P(\tau, \varepsilon) \cdot \tau d \tau . \\
H=2 \int_{0}^{\infty} \frac{\cos \tau^{2}}{I+e^{\frac{2 b}{x} \tau^{2}}}\{\cosh \tau \sqrt{2 \omega}-\cos \tau \sqrt{2 \omega}\} \tau d \tau
\end{gathered}
$$

avremo per $n$ la espressione:

$$
\left.n=\frac{a}{\pi x}[K+H]^{4}\right)
$$

4) Questa espressione di $\eta$ coincide sostanzialmente con la espressione datale in $M$.

Si osservi che per $b=\infty$ la espressione di $\eta$ si riduce a $\eta=\frac{a}{\pi x} K$. Come ho annunciato nella prefazione, questa espressione di $\eta$, relativa al canale infinitamente profondo, coincide in sostanza con la forma datale da CaUCHy. Credo non fuori di luogo mostrare, come si possa passare rigorosamente da questa espressione di $K$ a quella classica riportata in M. [form. (29), pag. (383)].

$\mathrm{Si}$ considerino le due identità :

$$
\begin{aligned}
\int_{1}^{\infty} \sin \frac{\omega}{2}\left(1-\tau^{2}\right) P\left(\sqrt{\frac{\omega}{2}}[\tau-\mathrm{I}], \varepsilon\right) d \tau & =\frac{1}{2} \int_{0}^{\infty} \sin \frac{\omega}{2}(1-\tau) P\left(\sqrt{\frac{\omega}{2}}[\sqrt{\tau}-1], \varepsilon\right) \frac{d \tau}{\sqrt{\tau}} \\
& -\frac{1}{2} \int_{0}^{1} \sin \frac{\omega}{2}(1-\tau) P\left(\sqrt{\frac{\omega}{2}}[\sqrt{\tau}-1], \varepsilon\right) \frac{d \tau}{\sqrt{\tau}}, \\
\int_{-1}^{\infty} \sin \frac{\omega}{2}\left(I-\tau^{2}\right) P\left(\sqrt{\frac{\omega}{2}}[\tau+1], \varepsilon\right) d \tau & =\frac{1}{2} \int_{0}^{\infty} \sin \frac{\omega}{2}(1-\tau) P\left(\sqrt{\frac{\omega}{2}}[\sqrt{\tau}+1], \varepsilon\right) \frac{d \tau}{\sqrt{\tau}} \\
& +\frac{1}{2} \int_{0}^{1} \sin \frac{\omega}{2}(1-\tau) P\left(\sqrt{\frac{\omega}{2}}[\sqrt{\tau}-1], \varepsilon\right) \frac{d \tau}{\sqrt{\tau}} .
\end{aligned}
$$


Il nostro compito è ora quello di asseguare un valore approssimato per gli integrali $K$ e $H$.

$\int$ II.

\section{Il metodo di Riemann.}

I. II metodo in generale, - Vogliasi calcolare il valore asintotico rispetto ad $\alpha$, di un integrale della forma:

$$
\int \lambda_{0}(\tau) e^{-x f(\tau)} d \tau
$$

il cammino di integrazione essendo l'asse reale $\tau$.

Supporremo $\alpha>0$.

Si interpreti $\tau$ come variabile complessa $\tau=\mu+i$ v e si indichino con $X$ e $Y$ la parte reale e immaginaria della funzione $f(\tau)$, cosicchè sia:

$$
f(\tau)=X+i Y
$$

Il pensiero di Riemann consiste in ciò: deformare il cammino di integrazione in modo, che lungo di esso $X$ prenda dei grandi valori positivi, per cui, in prima approssimazione, per $\alpha$ abbastanza grande, $e^{-\alpha X}$ è sensibilmente eguale a zero e basterà prendere in considerazione $i$ tratti del cammino di integrazione nella vicinanza dei punti nei quali $X$ assume $\mathrm{i}$ valori più piccoli.

Osserviamo, in primo luogo, che $X$ e $Y$, considerate come funzioni di $\mu$ e $v$, risultano notoriamente funzioni armoniche, in virtù della monogeneità, per cui non esisterà per esse alcun punto di massimo o di minimo. I punti per $\mathrm{i}$ quali

$$
\frac{\partial X}{\partial \mu}=0, \quad \frac{\partial X}{\partial v}=0
$$

Scambiando nei due integrali dei primi membri rispettivamente il $\tau$ in $\tau \sqrt{\frac{2}{\omega}}+$ I e $\tau \sqrt{\frac{2}{\omega}}-1$, considerandone poi $i$ limiti per $\varepsilon=\mathrm{I}$, [poichè $\lim _{\varepsilon=1} P=\mathrm{I}$ ], otteniamo:

$$
\begin{aligned}
& \lim _{\varepsilon=1} \sqrt{\frac{2}{\omega}} \int_{0}^{\infty} \sin \left(\tau^{2}+\tau \sqrt{2 \omega}\right) P(\tau, \varepsilon) d \tau=-\frac{I}{2} \int_{0}^{\infty} \sin \frac{\omega}{2}(I-\tau) \frac{d \tau}{\sqrt{\tau}}+\frac{I}{2} \int_{0}^{1} \sin \frac{\omega}{2}(I-\tau) \frac{d \tau}{\sqrt{\tau}}, \\
& \lim _{\varepsilon=1} \sqrt{\frac{2}{\omega}} \int_{0}^{\infty} \sin \left(\tau^{2}-\tau \sqrt{2 \omega}\right) P(\tau, \varepsilon) d \tau=-\frac{I}{2} \int_{0}^{\infty} \sin \frac{\omega}{2}(I-\tau) \frac{d \tau}{\sqrt{\tau}}-\frac{I}{2} \int_{0}^{I} \sin \frac{\omega}{2}(I-\tau) \frac{d \tau}{\sqrt{\tau}} .
\end{aligned}
$$

Sottraendo ora la $\mathrm{II}^{\mathrm{a}}$ dalla $\mathrm{I}^{\mathrm{a}}$, con un ovvio cambiamento di variabile nel secondo membro, viene:

$$
\lim _{\varepsilon=1} 2^{3 / 2} \int_{0}^{\infty} \cos \tau^{2} \sin \tau \sqrt{2 \omega} P(\tau, \varepsilon) d \tau=\int_{0}^{\omega} \sin \frac{I}{2}(\omega-\tau) \frac{d \tau}{\sqrt{\tau}}
$$

ed eseguendo una derivazione rispetto ad $\omega$ :

$$
K=\frac{\sqrt{\omega}}{2} \int_{0}^{\omega} \cos \frac{\mathrm{I}}{2}(\omega-\tau) \frac{d \tau}{\sqrt{\tau}}
$$


sono punti di sella per $X$ e quindi anche per $Y$ e vengono quindi determinati dalla equazione :

$$
\frac{d f(\tau)}{d \tau}=0
$$

Prendiamo ora a considerare la superficie [che immagineremo proiettata sul piano $(u, v)]$

$$
X=X(\mu, \nu)
$$

e determiniamo le linee di massima pendenza per $X$.

Se $s$ è la linea lungo la quale $X$ cresce il più rapidamente possibile ed $n$ è la sua normale, lungo di essa dovrà essere:

$$
\frac{d X}{d n}=0
$$

e poichè $\frac{d X}{d n}=\frac{d Y}{d s}$, la linea di massima pendenza $s$ sarà determinata dall'equazione:

$$
Y=\text { cost. }
$$

Fra tutte le possibili linee di integrazione (II) noi dovremo scegliere quella lungo la quale $X$ conserva sempre valore positivo; ed inoltre, se osserviamo che le linee (II) devono uscire dai punti dove $X$ diviene infinito (per il fatto che $X$ non ammette nè massimo, nè minimo finito), se ne inferisce che le linee che ci interessano devono passare per un punto di sella e riescono percio perfettamente individuate da queste condizioni.

Una volta determinata la linea $\mathrm{Y}=$ cost., si muterd il cammino originario di integrazione (tenendo conto delle eventuali singolarità della funzione integranda), assumendo come nuovo cammino la linea s. Poi, in generale, basterd limitare l'integrazione a un piccolo intorno del punto di sella. Per il successo del metodo è necessario poi che l'ampiezza dell'intervallo d'integrazione sia piccola in modo, che in esso si possa ritenere nulla (in prima approssimazione) l'oscillazione della funzione $\lambda(\tau)$.

Notiamo subito che è pure evidentemente necessario che il cammino di integrazione originario e quello deformato possano costituire una linea chiusa, magari, se vogliamo, con l'aggiunta di tratti, i quali però non rechino contributo alcuno al valore dell'integrale. Questo fatto giustifica la rilevata necessità di mutare la primitiva espressione di $n$, datale nella mia precedente Memoria.

2. Esame della funzione $f(\tau)=i\left\{\tau^{2}-\tau \sqrt{2 \omega} \overline{0}\right.$. - Supponiamo, che la funzione $f(\tau)$ del $\mathrm{n}^{\circ}$ precedente sia della forma:

con $\omega$ costante reale positiva.

$$
f(\tau)=i\left\{\tau^{2}-\tau \sqrt{2 \omega}\right\}
$$

Posto

risulterd

$$
\tau=\mu+i v
$$

$$
\begin{aligned}
& X=-2 \mu \nu+\nu \sqrt{2 \omega}, \\
& Y=\mu^{2}-\nu^{2}-\mu \cdot \sqrt{2 \omega} .
\end{aligned}
$$


Le regioni nelle quali la funzione $X$ è positiva, sono separate da quelle in cui è negativa, dalla curva $X=0$, che si scompone evidentemente nelle due rette:

$$
\nu=o, \quad \mu=\sqrt{\frac{\omega}{2}} .
$$

Immediatamente si constata allora che le regioni nelle quali la funzione $X$ é sempre

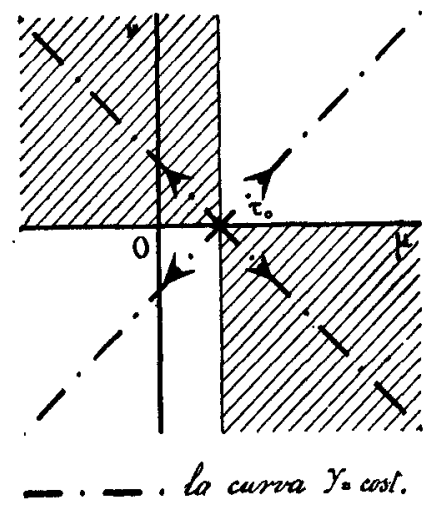

(Fig. I).

positiva sono determinate dalle diseguaglianze:

$$
\begin{array}{ll}
\nu>0, & \mu<1 \sqrt{\frac{\omega}{2}}, \\
\nu<0, & \mu>1 \sqrt{\frac{\omega}{2}} .
\end{array}
$$

Nella Fig. I tali regioni sono tratteggiate.

L'equazione $\frac{d f(\tau)}{d \tau}=\mathrm{o}$ ha l'unica radice $\tau=\sqrt{\frac{\omega}{2}}$, quindi per la nostra funzione esiste il solo punto di sella $\tau_{\mathrm{o}}=\sqrt{\frac{\omega}{2}}$, punto che giace sull'asse reale.

Tra le curve $Y=$ cost., quella che passa per il punto $\tau_{0}$, ha allora per equazione:

$$
\mu^{2}-v^{2}-\mu \sqrt{2 \omega}+\frac{\omega}{2}=0
$$

che si scompone nelle due seguenti:

$$
\begin{aligned}
& \mu+\nu=\sqrt{\frac{\omega}{2}}, \\
& \mu-v=\sqrt{\frac{\omega}{2}} .
\end{aligned}
$$

Si tratta quindi di due rette inclinate di $45^{\circ}$ sugli assi coordinati, di cui la prima 
corre tutta nella regione dove $X$ è positivo e costituirà quindi la linea di integrazione, che per noi avrà speciale interesse.

Lungo di essa $X$ cresce il più rapidamente possibile a partire dal punto di sella $\tau_{0}=\sqrt{\frac{\omega}{2}}$, la direzione di pendenza essendo indicata dalle freccie (Fig. I).

$\Im$ III.

Trasformazione della espressione di $n$.

I. Trasformazione degll integrali $K$ e $H$. - Prendiamo ora a considerare gli integrali $K$ e $H$, che compariscono nell'espressione di $n$ [form. (Io)] e mettiamoli sotto la forma seguente:

$$
\begin{gathered}
K=\lim _{\varepsilon=1} \int_{0}^{\infty} \cos \left(\tau^{2}-\tau \sqrt{2 \omega}\right) P(\tau, \varepsilon) \tau d \tau+\lim _{\varepsilon=1} \int_{0}^{\infty} \cos \left(\tau^{2}+\tau \sqrt{2 \omega}\right) P(\tau, \varepsilon) \tau d \tau \\
H=H_{2}-H_{1}
\end{gathered}
$$

dove abbiamo posto:

$$
\begin{aligned}
& H_{1}=\int_{0}^{\infty} \frac{\cos \left(\tau^{2}-\tau \sqrt{2 \omega}\right)}{\mathrm{I}+e^{\frac{2 h}{x} \tau^{2}}} \tau d \tau+\int_{0}^{\infty} \frac{\cos \left(\tau^{2}+\tau \sqrt{2 \omega}\right)}{\mathrm{I}+e^{\frac{2 h}{x} \tau^{2}}} \tau d \tau \\
& H_{2}=\int_{i \infty}^{\infty} \frac{\cos \left(\tau^{2}-\tau \sqrt{2 \omega}\right)}{\mathrm{I}+e^{-\frac{2 h}{x} \tau^{2}}} \tau d \tau+\int_{i \infty}^{\infty} \frac{\cos \left(\tau^{2}+\tau \sqrt{2 \omega}\right)}{\mathrm{I}+e^{-\frac{2 h}{x} \tau^{2}} \tau d \tau}
\end{aligned}
$$

Poichè $x$, per ipotesi, è positivo ( $\mathrm{cfr} . ~ \ \mathrm{I}, \mathrm{n}^{\mathrm{o}} 4$ ) ed essendolo $t$ per la sua natura, le prime "parti di questi integrali $K, H_{1}$ ed $H_{2}$ rappresentano la perturbazione, che si propaga nella direzione delle ascisse positive, mentre le altre parti rappresentano la perturbazione che si propaga nel verso opposto.

Noi prenderemo a considerare la perturbazione, per cosi dire, positiva: potrcmo quindi scrivere di conseguenza:

$$
\begin{gathered}
\left.K=\lim _{\varepsilon=1} R \int_{0}^{\infty} e^{-i\left(\tau^{2}-\tau v^{-} \omega\right.}\right) P(\tau, \varepsilon) \tau d \tau, \\
H_{t}=R \int_{0}^{\infty} \frac{e^{-i\left(\tau^{2}-\tau / \sqrt{2 \omega}\right)} \tau d \tau}{\mathrm{I}+e^{\frac{2 b}{x} \tau^{2}}}, \\
H_{2}=R \int_{i \infty}^{\infty} \frac{e^{-i\left(\tau^{2}-\tau \sqrt{ } / \overline{2}\right)} \tau d \tau}{\mathrm{I}+e^{-\frac{2 b}{x} \tau^{2}}}
\end{gathered}
$$

il significato della lettera $R$ essendo manifesto. 
2. Una prima modificazione del cammino di inteśazione. - Interpretiamo ora $\tau$ come variabile complessa, ponendo:

$$
\tau=\mu+i v
$$

e fissiamo, in primo luogo, la nostra attenzione sull'integrale $K$.

Consideriamo nel piano $(\mu, v)$ il cammino chiuso $O A B C$, formato dai lati di un rettangolo, la cui posizione é individuata dalla figura accanto, con la condizione però

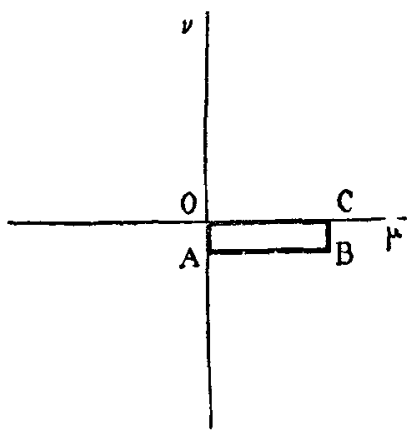

(Fig. 2).

che non racchiuda alcun polo della funzione $\frac{I}{I+e^{\frac{2 h}{x} \tau^{2}}}$.

Per il $I^{0}$ teorema di CaUChy, ponendo per brevitd

sara

$$
f(\tau)=i\left(\tau^{2}-\tau \sqrt{2 \omega}\right)
$$

od anche

$$
\int_{O A B C} e^{-f(\tau)} P(\tau, \varepsilon) \tau d \tau=0
$$

$$
\int_{O C}=\int_{O A B}+\int_{B C}
$$

Facciamo ora crescere indefinitamente $\mathrm{i}$ lati $O C$ ed $A B$ del rettangolo ${ }^{5}$ ). In base allo studio fatto precedentemente della funzione $f(\tau)=i\left(\tau^{2}-\tau \sqrt{2 \omega}\right)\left(\mathrm{cfr} . \$ \mathrm{II}, \mathrm{n}^{\circ} 2\right)$, potremo affermare che al limite la parte $B C$ dà contributo nullo, per cui avremo:

$$
\int_{0}^{\infty} e^{-f(\tau)} P(\tau, \bar{\varepsilon}) \tau d \tau=\int_{(1)} e^{-f(\tau)} P(\tau, \varepsilon) \tau d \tau
$$

il cammino (I) essendo costituito per un piccolo tratto dall'asse imaginario negativo $v$ e poi da una parallela all'asse reale $\mu$, fino all'infinito.

5) $\mathrm{Si}$ osservi che in tal modo la striscia di piano che si viene ad ottenere non racchiuderà mai alcun polo della funzione $\frac{I}{I+e^{\frac{2 h}{x} \tau^{2}}}$ per l'ipotesi enunciata che non ne racchiuda il rettangolo $O A B C$. 
E poichè lungo il cammino (I) la funzione $e^{-f(\tau)}$ si mantiene integrabile anche all' $\infty$ ed è

$$
\lim _{\varepsilon=1} P(\tau, \varepsilon)=I
$$

avremo:

$$
K=\lim _{\varepsilon=\mathrm{I}} R \int_{0}^{\infty} e^{-f(\tau)} P(\tau, \varepsilon) \tau d \tau=\int_{(1)} e^{-f(\tau)} \tau d \tau .
$$

3. Espressione the $n$ assume di conformità. -- Pensiamo anche negli integrali $H_{1}$ ed $H_{2}, \tau$ come variabile complessa. Potremo allora deformare il cammino di $H_{1}$, che originariamente va da o all' $\infty$, nel cammino (I) ed avremo:

Scriviamo poi :

$$
H_{1}=R \int_{(\mathrm{s})} \frac{e^{-f(\tau)} \tau d \tau}{\mathrm{I}+e^{\frac{2 h}{x} \tau^{2}}}
$$

$$
H_{2}=R \int \frac{e^{-f(\tau)} \tau d \tau}{\mathrm{I}+e^{-\frac{2 h}{x} \tau^{2}}}
$$

questo integrale essendo esteso a tutto l'asse immaginario positivo.

Ed ora essendo:

sarà :

$$
K-H_{\mathrm{t}}=R \int_{(\mathrm{l})} \frac{e^{-f(\tau)} \tau d \tau}{1+e^{-\frac{2 h}{x} \tau^{2}}}
$$

$$
K+H=K+H_{2}-H_{1}=R \int_{(2)} \frac{e^{-f(\tau)} \tau d \tau}{\mathrm{x}+e^{-\frac{2 h}{x} \tau^{2}}}
$$

il cammino (2) coincidendo con l'asse immaginario da $i \infty$ fino ad un punto al di sotto dell'origine $O$ e poi con una parallela all'asse reale fino all' $\infty$. Esso è indicato nella Fig. 3: le freccie indicano il verso di percorrenza.

Ne consegue che, a norma della (10), ad $n$ compete la espressione

$$
n=\frac{a}{\pi x} R \int_{(2)} \frac{e^{-f(\tau)} \tau d \tau}{1+e^{-\frac{2 h}{x} \tau^{2}}} .
$$

\section{\IV.}

\section{Applicazione del metodo di Riemann.}

1. II cammino definitivo (3). - A noi interessa ora deformare ulteriormente il cammino di integrazione (2) in modo da poter applicare il metodo di Riemans.

Per quanto abbiamo esposto nel $\$ II, e per lo studio fatto della funzione $f(\tau)$, 
possiamo affermare che il nuovo cammino, lungo il quale conviene estendere il nostro integrale, è costituito dalla retta:

$$
\mu+\nu=\sqrt{\frac{\omega}{2}}
$$

cammino, che chiameremo (3) e che potrd̀ esser immaginato congiunto all'infinito col cammino (2), in modo da costituire con esso una linea chiusa. Notiamo subito che il cammino ideale che congiunge $i$ due cammini (2) e (3) non reca contributo alcuno al valore del nostro integrale.

È necessario ora tener presente, che la funzione integranda non si mantiene sempre finita e precisamente ammette dei poli nei punti:

$$
\tau_{p}= \pm(\mathrm{I} \pm i) \frac{\mathrm{I}}{2} \sqrt{\frac{x}{b}(2 p+\mathrm{I}) \pi} \quad(p=0,1,2, \ldots) .
$$

Potrd quindi avvenire che $i$ due cammini (2) e (3) non racchiudano nel loro in. terno alcun polo, oppure ne racchiudano un certo numero. Se poniamo:

$$
\Theta=\frac{t \sqrt{g b}}{2 x}
$$

si constata immediatamente che il primo caso avverrà quando $\theta<\sqrt{\pi}$ ed il secondo quando $\Theta>\sqrt{\pi}$. E chiaro poi che vi sono degli istanti in cui il cammino (3) passa proprio attraverso un polo e precisamente quando $\Theta=\sqrt{(2 p+\mathrm{I}) \pi}$.

Nel caso in cui $\Theta<\sqrt{\pi}$ il cammino (2) si potrd deformare semplicemente nel

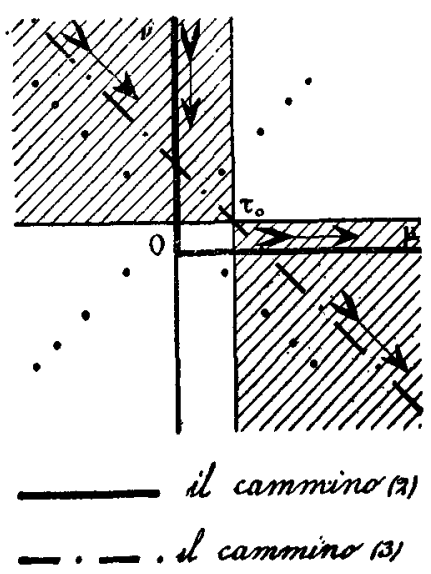

(Fig. 3).

cammino (3); nel $\Pi^{\circ}$ caso bisognerà invece tener conto dei poli a norma del II ${ }^{\circ}$ teorema di CaUCHY. 
Avremo quindi per $n$ la espressione:

$$
n=\frac{a}{\pi x} R\left[\int_{(3)} \frac{e^{-f(\tau)} \tau d \tau}{\mathrm{I}+e^{-\frac{2 h}{x} \tau^{2}}}+2 \pi i \sum T\right]
$$

$\sum T$ essendo la somma dei residui relativi agli eventuali poli contenuti nell'interno del contorno (2), (3).

2. Valore asintotico dell'integrale $\int_{(3)} \frac{e^{-f(t)} \tau d \tau}{I+e^{-\frac{2 h}{x} \tau^{2}}} \cdot$ - Occupiamoci ora finalmente del valore asintotico di questo integrale.

Poichè è identicamente:

sard

$$
f(\tau)=i\left\{\tau^{2}-\tau \sqrt{2 \omega}\right\}=i\left\{-\frac{\omega}{2}+\left(\tau-\sqrt{\frac{\omega}{2}}\right)^{2}\right\}
$$

$$
\int_{(3)} \frac{e^{-f(\tau)} \tau d \tau}{\mathrm{I}+e^{-\frac{2 h}{x} \tau^{2}}}=e^{i \frac{\omega}{2}} \int_{(3)} \frac{e^{-i\left(\tau-\sqrt{\frac{\omega}{2}}\right)^{2}} \tau d \tau}{\mathrm{I}+e^{-\frac{2 h}{x} \tau^{2}}}
$$

Possiamo ora in quest'ultimo integrale cambiare la variabile d'integrazione, mediante la posizione:

$$
\tau-\sqrt{\frac{\omega}{2}}=\sqrt{\omega} e^{-i \frac{\pi}{4}} \xi
$$

purchè si abbia l'avvertenza di estendere il nuovo integrale da $-\infty$ a $+\infty$ e ne segue quindi :

con

$$
\int_{(3)} \frac{e^{-f(\tau)} \tau d \tau}{I+e^{-\frac{2 h}{x} \tau^{2}}}=e^{i\left(\frac{\omega}{2}-\frac{\pi}{4}\right)} \cdot \frac{\omega}{\sqrt{2}} \cdot I
$$

$$
I=\int_{-\infty}^{\infty} \frac{e^{-\omega \xi^{2}}\left(\mathrm{I}+\xi e^{-i \frac{\pi}{4}} \sqrt{2}\right) d \xi}{1+e^{-\frac{b}{x} \omega\left(1+\xi e^{-i \frac{\pi}{4}} \sqrt{2}\right)^{2}}} .
$$

Questo integrale va ora calcolato nella immediata vicinanza del punto di sella $\tau_{\mathrm{o}}=\sqrt{\frac{\omega}{2}}$, ossia $\xi=0$, quindi sard:

avendo posto:

$$
I=\int_{-1}^{\varepsilon} e^{-\omega \xi^{2}} \lambda(\xi) d \xi
$$

$$
\lambda(\xi)=\frac{1+\xi e^{-i \frac{\pi}{4}} \sqrt{2}}{1+e^{-\frac{b}{x} \omega\left(1+\xi e^{\left.-i \frac{\pi}{4} \sqrt{2}\right)^{2}}\right.} .}
$$

Ora affinchè il metodo sia ammissibile è necessario che $e^{-\omega t^{2}}$ sia praticamente nullo, 
il che avviene, trascurando la terza cifra decimale, quando

ossia

$$
\omega \varepsilon^{2} \supseteq 5
$$

$$
\varepsilon \geqslant \sqrt{\frac{5}{\omega}}
$$

Ma d'altra parte per il successo del metodo si richiede altresì che $\varepsilon$ sia abbastanza piccolo, perchè si possa ritenere trascuratile fra $-\varepsilon$ e $+\varepsilon$ l'oscillazione della funzione $\lambda(\xi)$. Una tale oscillazione dipende manifestamente dai parametri che compariscono in $\lambda$, in particolare da $\omega$, e dall'ampiezza $\varepsilon$. Designandola, per un momento, con $\Delta(\varepsilon, \omega)$, dovremo ottemperare alla condizione

$$
\Delta(\varepsilon, \omega)<\frac{I}{n}
$$

assumendo, ad esempio, $n=$ Ioo.

Per $\omega=\infty$, la (I5) si riduce ad $\varepsilon>0$ ed inoltre si ha $\Delta=2 \sqrt{2} \varepsilon$. Essendo allora la funzione $\lambda$ continua nellintervallo $(-\varepsilon, \varepsilon)$, si vede che vi è un ambito di valori per $\omega$ ed $\varepsilon$ che rendono soddisfatte contemporaneamente le due condizioni (I5) e (16).

In prima approssimazione possiamo quindi ritenere il tattore $\lambda(\xi)$ come costante ed eguale al valore che assume nel punto di sella $\zeta=0$, perció non rimane da valutare che l'integrale $\int_{-\varepsilon}^{\varepsilon} e^{-\omega \xi^{2}} d \xi$, che ha praticamente lo stesso valore come se $\mathrm{i}$ limiti fossero $\pm \infty$. E poichè

$$
\int_{-\infty}^{\infty} e^{-\omega \xi^{2}} d \xi=\sqrt{\frac{\pi}{\omega}}
$$

cosi risulta che asintoticamente è:

$$
I=\frac{\mathrm{I}}{\mathrm{I}+e^{-\frac{b}{x} \omega}} \sqrt{\frac{\pi}{\omega}} .
$$

3. Contributo dovuto ai poli. $-\mathrm{Al}$ crescere di $\omega$ il punto di sella si muove lungo l'asse reale allontanandosi dall'origine. Ne segue che dopo un certo istante, $i$ due cammini (2) e (3) racchiudono, come abbiamo notato, un certo numero di poli della funzione $\frac{1}{I+e^{-\frac{2 b}{x} \tau^{2}}}$, poli che sono dati dalla formula

$$
\tau_{p}=(\mathrm{I}+i) \frac{\mathrm{I}}{2} \sqrt{\frac{x}{b}(2 p+\mathrm{I}) \pi} \quad(p=0,1,2, \ldots) .
$$

Il residuo che proviene da un generico polo $\tau_{p}$ si calcola con tutta facilita e si trova :

$$
T=\frac{x}{4 b} e^{(2 p+1) \frac{\pi x}{2 h}-(1-i) \sqrt{\frac{\omega x}{2 h}(2 p+1) \pi}} .
$$


Il tc:mine $\sum T$ che comparisce nella (13) sarà quindi del tipo:

$$
\sum T=\frac{x}{4 b} \sum_{0} e^{(2 p+1) \frac{\pi x}{2 h}-(1-i) \sqrt{\frac{\omega x}{2 h}(2 p+1) \pi}}
$$

la somma constando di tanti termini quanti sono $\mathrm{i}$ poli che cadono tra $\mathrm{i}$ cammini (2) e (3).

4. Equazione del pelo libero. - Ed ora, mediante le (I4) e (I7), la formula (I3) diventa :

$$
n=\frac{a}{\pi x} R\left[e^{i\left(\frac{\omega}{2}-\frac{\pi}{4}\right)} \sqrt{\frac{\omega \pi}{2}} \frac{\mathrm{I}}{\mathrm{I}+e^{-\frac{b}{x} \omega}}+2 \pi i \sum T\right]
$$

da cui, richiamando la (I8) e prendendo la parte reale :

(19) $n=\frac{a \sqrt{\omega}}{x \sqrt{2 \pi}} \cos \left(\frac{\omega}{2}-\frac{\pi}{4}\right) \frac{\mathrm{I}}{\mathrm{I}+e^{-\frac{b}{x} \omega}}-\frac{a}{2 b} \sum_{0} e^{(2 p+1) \frac{\pi x}{2 b}-\sqrt{\frac{\omega x}{2 b}(2 p+1) \pi}} \sin \sqrt{\frac{\omega x}{2 b}(2 p+1) \pi}$.

Non sard fuori di luogo notare anche qui, che facendo tendere $b$ all' $\infty$, si ottiene la classica formula :

$$
\left.n=\frac{a \sqrt{\omega}}{x \sqrt{2 \pi}} \cos \left(\frac{\omega}{2}-\frac{\pi}{4}\right)^{6}\right) \text {. }
$$

Se ci limitiamo a considerare il caso in cui $\Theta=\frac{t \sqrt{g h}}{2 x}<\sqrt{\pi}$, allora la (19) si riduce semplicemente alla:

$$
n=\frac{a \sqrt{\omega}}{x \sqrt{2 \pi}} \cos \left(\frac{\omega}{2}-\frac{\pi}{4}\right) \frac{\mathrm{I}}{\mathrm{I}+e^{-\frac{b}{x} \omega}} .
$$

Poniamo

$$
c_{\mathrm{o}}=\sqrt{g b}
$$

e fissiamo la nostra attenzione sull'andamento del fenomeno in un certo punto di

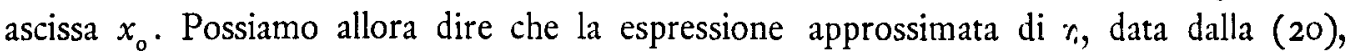
va bene finchè

$$
t<2 \sqrt{\pi} \frac{x_{0}}{c_{0}}
$$

Notiamo che $c_{\mathrm{o}}$ è la velocità con cui si propagano le onde lunghe in regime costante, quindi $\frac{x_{0}}{c_{0}}$ è il tempo che impiega un'onda lunga a raggiungere la posizione $x_{0}$.

$\mathrm{Ne}$ segue che la (20) rappresenta con sufficiente approssimazione la configurazione del pelo libero nel posto $x_{0}$, per un lasso di tempo eguale a circa 3 volte e mezza il tempo impiegato a raggiungere la posizione prefissata dalle onde più lunghe.

Se chiamiamo segnale l'onda che col suo arrivo ci avverte non esser più valida la (20), vediamo che il segnale si propaga con velocita $\frac{c_{0}}{2 \sqrt{\pi}}$.

6) Cfr. H. LAMB, Lebrbuch der Hydıodynamik (traduzione di FRIEDEL) [Leipzig, Teubner, 1907], pag. $45 \mathrm{I}$, form. (22). 
Nell'istante in cui è proprio $\Theta=\sqrt{\pi}$ abbiamo un punto critico in cui l'equazione del pelo libero cambia repentinamente di forma, perchè al secondo membro della (20), bisogna aggiungere un nuovo termine.

Il nuovo segnale si propaga con velocitì $\frac{c_{o}}{2 \sqrt{3 \pi}}$ e nell'istante in cui è $\Theta=\sqrt{3 \pi}$ abbiamo un nuovo punto critico.

Constatiamo cosi la presenza di tanti segnali all'arrivo di ciascuno dei quali, in un dato posto, corrisponde un brusco cambiamento di forma nel pelo libero. Questi segnali si propagano con velocità sempre minore, in modo che sempre maggiore è il tempo che trascorre tra un cambiamento di forma e l'altro.

\section{SV.}

\section{Il metodo di Lord Kelvin.}

Ho gia accennato nella prefazione di M. al metodo di gruppo o della fase stazio. naria dovuto a LoRD KeLvin e che ha per iscopo la determinazione della parte principale di una perturbazione ondosa rappresentata da un integrale definito.

LoRd Kelvin fu condotto alla scoperta del metodo, che porta il suo nome, dalla penetrante intuizione del modo con cui si svolgono i fenomeni fisici, intuizione che contrassegna in modo particolare i grandi matematici inglesi. Il metodo stesso, spogliato della sua veste fisica, porge il modo di calcolare il valore asintotico di alcuni integrali definiti.

Il prof. Haveloch, che espone il metodo nel suo piccolo trattato su "The propagation of disturbances in dispersive media " ${ }^{7}$ ) ne fa parecchie applicazioni nello studio della propagazione delle onde in un mezzo dispersivo, ma dà nell'ultimo capitolo degli esempi in cui il metodo stesso non puó esser applicato.

Il mio primo pensiero, nell'intraprendere le ricerche che formano l'oggetto della presente Memoria, fu quello di applicare il metodo di Kelvin allo studio asintotico degli integrali che ho denotato con $K, H_{1}$ ed $H_{2}$. Il metodo riesce per gli integrali $K$ ed $H_{1}$, ma non è applicabile per l'integrale $H_{2}$, ragione per cui ho dovuto abbandonare l'idea.

Il metodo di KeLvin applicato agli integrali $K$ ed $H_{1}$ porge per in la espressione (20). Val quindi la pena di rilevare che anche col metodo di Kelvin si raggiunge una sufficiente approssimazione col tener conto solamente degli integrali $K$ ed $H_{1}$ e trascurando completamente l'integrale $\mathrm{H}_{2}$.

Padova, novembre 1915.

Attilio Palatini.

7) Cambridge: at the University Press, I9I4. 